\title{
Facile Synthesis of Fe-Doped Titanate Nanotubes with Enhanced Photocatalytic Activity for Castor Oil Oxidation
}

\author{
Guozhu Fu, Gang Wei, Yanqiu Yang, WeiCheng Xiang, and Ning Qiao \\ State Key Laboratory of Chemical Resource Engineering, Beijing University of Chemical Technology, Beijing 100029, China \\ Correspondence should be addressed to Ning Qiao; qiaoning@mail.buct.edu.cn
}

Received 13 December 2012; Accepted 31 December 2012

Academic Editor: Shao-Wen Cao

Copyright ( 2013 Guozhu Fu et al. This is an open access article distributed under the Creative Commons Attribution License, which permits unrestricted use, distribution, and reproduction in any medium, provided the original work is properly cited.

Iron-doped titanate nanotubes were synthesized by hydrothermal method, and the photocatalytic activity was greatly enhanced by iron doping.

Followed by the discovery of carbon nanotubes, synthesis of one-dimensional (1D) nanomaterials has attracted great interest because of their exceptional electrical and mechanical properties [1-4]. Some inorganic $1 \mathrm{D}$ nanomaterials including $\mathrm{ZnO}, \mathrm{VO}_{x}$, and $\mathrm{TiO}_{2}$ have been synthesized in recent years [5-8]. Among these materials, titanic compound nanotubes have stimulated particular interest. Titanic nanocrystals have been extensively studied in photocatalytic or photoelectrochemical systems and so forth [9-12], and fabrication of tubular structures offers an effective approach to adjust their properties, which are crucial in practical applications. For example, the photocatalytic activity of $\mathrm{TiO}_{2}$ could be enhanced by the tubular structures because of their large specific surface, which leads to a higher potential of applications in environmental purification and generation of hydrogen gas and so forth [13].

Recently, particular interest is devoted to obtain $\mathrm{H}_{2} \mathrm{Ti}_{3} \mathrm{O}_{7}$-type nanotubes synthesized by hydrothermal method [14-16], and these nanotubes show excellent ionexchange ability and photocatalytic activities and may be applied to photocatalysis, photoluminescence, and dyesensitized solar cells [3]. However, their structures are still not well understood. The photocatalytic property is originated from the charge carriers produced by the excitation process on the particle surface, and the photocatalytic efficiency is determined by the transfer rate and recombination rate of carriers [17]. However, the carriers are usually unstable and easy to recombine. To improve the photocatalytic efficiency, the transfer rate must be enhanced and recombination rate should be reduced. Introducing other elements especially the transition metal ions into the matrix has been proved to be an effective method to improve the photocatalytic efficiency, and many reports on the doped $\mathrm{TiO}_{2}$ and their properties have been published [17-19]. More recently, followed by the development of researches on one-dimensional materials, fabrication of doped nanorods and nanowires with transition metal ions has stimulated much interest due to their exclusive properties and potential applications [20-22]. However, doped tubelike nanostructures have never been reported. Since the tube channel has potential advantage to provide direct conduction paths for the electrons [23], it is reasonable to believe that doped tubelike structures will induce some excellent properties for their applications.

In this communication we reported that the Fe-doped titanated nanotubes (TiNTs) can be easily synthesized via a hydrothermal process. Commercial anatase $\mathrm{TiO}_{2}$ powders $(2 \mathrm{~g})$ and $\mathrm{FeCl}_{2} \cdot 4 \mathrm{H}_{2} \mathrm{O}(0.05 \mathrm{~g}, 0.15 \mathrm{~g}$, and $0.25 \mathrm{~g})$ were dispersed into an aqueous solution of $\mathrm{NaOH}\left(10 \mathrm{~mol} \mathrm{dm}^{-3}\right)$ and then moved into a Teflon-lined autoclave. The autoclave was heated in an oven at $130^{\circ} \mathrm{C}$ for $72 \mathrm{~h}$. The precipitate was filtrated and washed with diluted $\mathrm{HCl}$ until $\mathrm{pH}=7$. Final products were obtained by air-drying.

The structure analysis of obtained products was investigated by an X-ray diffraction (Philips X'Pert PRO MPD) operated at $40 \mathrm{kV}$ and $30 \mathrm{~mA}$ using $\mathrm{Cu} K \alpha$ radiation through the $2 \theta$-range from 5 to 70 degrees. The typical XRD pattern of products with various levels of iron doping is presented in Figure 1. In all cases, several weak and broad diffraction 


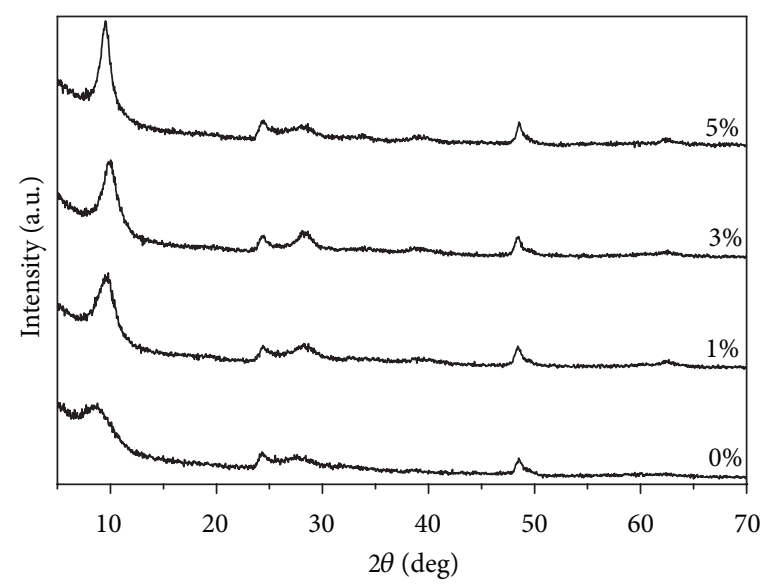

FIGURE 1: XRD pattern of synthesized sample.

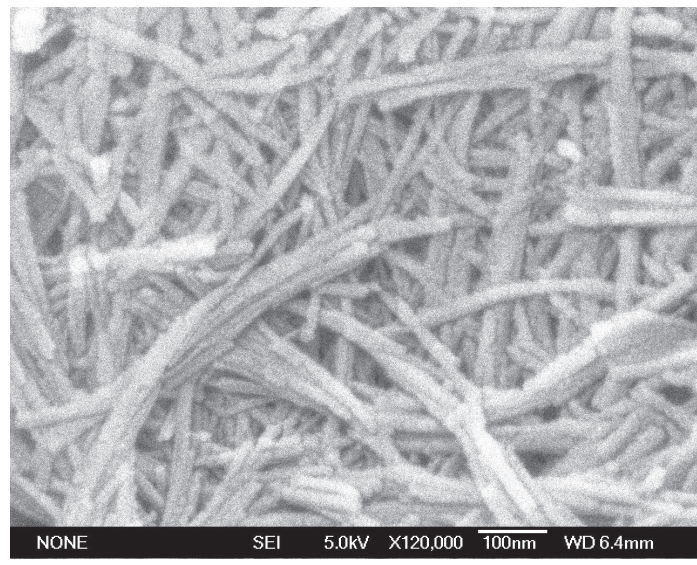

(a)

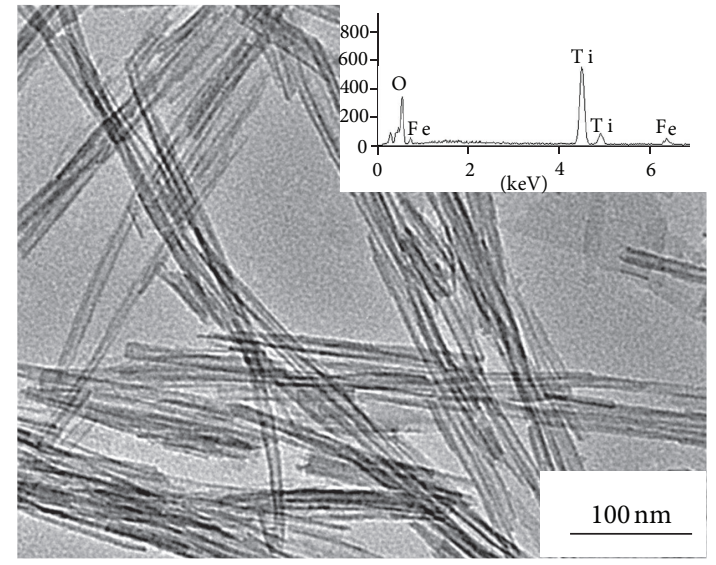

(b)

FIGURE 2: (a) SEM and (b) TEM images of synthesized products.

peaks exist (positioned at $2 \theta=9.9,24,28$, and $48^{\circ}$ ), which could be assigned to the diffraction peaks of titanates such as $\mathrm{H}_{2} \mathrm{Ti}_{3} \mathrm{O}_{7}$ structure (monoclinic unit cell with $a=1.603$, $b=0.375, c=0.919 \mathrm{~nm}$, and $\beta=101.45^{\circ}$ ) [13]. No crystalline anatase $\mathrm{TiO}_{2}$ or ferric oxide was detected in the pattern. In addition, the height of diffraction peaks is increased by the iron doping, which illustrates that the iron doping gives rise to the increase of the crystallization. Wang et al. [17] have reported that iron doping promotes the formation of rutile phase in $\mathrm{TiO}_{2}$ particles, while the influence of iron on the crystallization of titanate has never been reported.

Morphology of synthesized products was characterized by both field-emission scanning electron microscope (SEM, JSM-6700F) and transmission electron microscope (TEM, Hitachi H-800). Figure 2 gives the typical images of titanates with $\mathrm{R}_{\mathrm{Fe} / \mathrm{Ti}}=3 \%$. From the SEM micrograph shown in Figure 2(a) we can see that needle-shaped products with uniform morphology are obtained. The typical diameter and length are about 20 and $300 \mathrm{~nm}$, respectively, and no particles or layered structures were observed from the image. The
TEM image shown in Figure 2(b) reveals the nanotubular structures of synthesized products. A large quantities of tubular nanoparticles with uniform diameter about $20 \mathrm{~nm}$ were synthesized, and the length of obtained particles is about several hundred nanometers.

The energy dispersive X-ray spectroscopy (EDX) analysis recorded from the synthesized nanotubes illustrates that the characteristic peaks of $\mathrm{Fe}, \mathrm{Ti}$, and $\mathrm{O}$ were detected, indicating that Fe ions were successfully doped into the lattice of titanate.

Raman spectrum of synthesized nanotubes with different iron levels is shown in Figure 3. They are almost identical except for the intensity of peaks. The Raman features of synthesized nanotubes could be roughly regarded as a reflection of the six-coordinated layered titanate although the iron was doped into the titanate [3], but the exact assignment of the Raman spectra to specific active modes in layered titanates is still not well understood. In addition, these peaks are broadened and strengthened with increasing the iron levels, which reflects the split of lattice vibration modes caused by the decrease of symmetry. Raman spectroscopy is widely used 


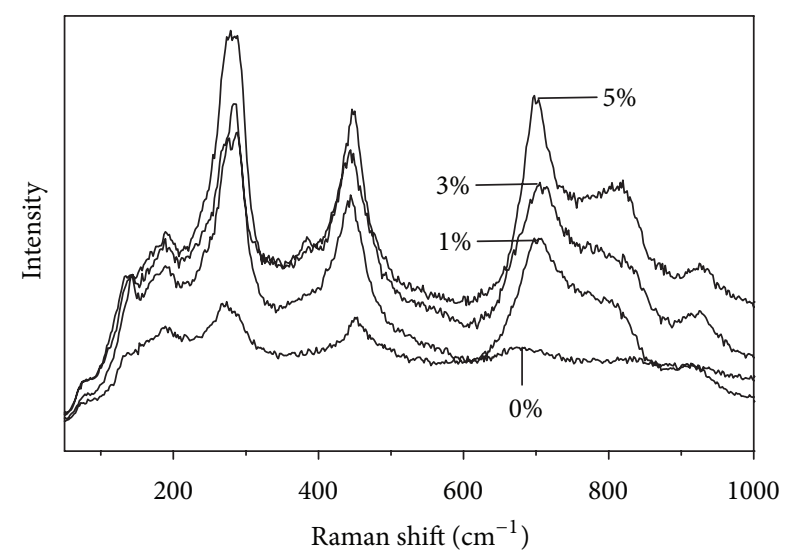

FIGURE 3: Raman spectra of synthesized sample.

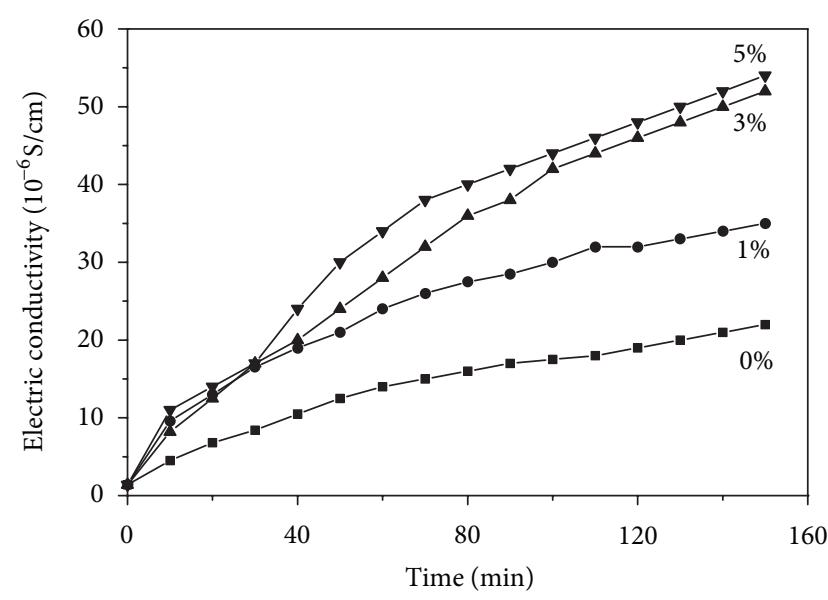

FIGURE 4: Photocatalytic activities of synthesized nanotubes.

to investigate the near-surface defect structure because of its surface sensitiveness, accordingly, the increase of peak height could be attributed to the increase of oxygen vacancies in the titanates. The defects promote charge transfer and efficiently separate the electrons and holes by shallow trapped electrons, which might give rise to remarkable increase of photocatalytic activity.

Photocatalytic activities of synthesized nanotubes were evaluated by the conductometric determination method $(\mathrm{CDM})$ which uses castor oil as probe reactant $[24,25]$. It has been confirmed that this method is effective for estimating the catalytic activity of inorganic pigments in oil cosmetics. In the test, $0.2 \mathrm{~g}$ of synthesized TiNTs was mixed with $20 \mathrm{~mL}$ of castor oil and moved into a quartz tube with UV-light irradiation for $150 \mathrm{~min}$. Air gas was bubbled in and then flowed out and induced into the deionized water placed in an electric conductivity measurement cell. Volatile molecules produced by the oxidation of the castor oil were trapped in the water by the effluent gas leading to the increase of conductivity. The degree of photocatalytic activity was estimated by the extent of conductivity change.
Figure 4 shows the photocatalytic activities of synthesized nanotubes, and we can conclude from the results that the photocatalytic activities are greatly enhanced by the iron doping. The change curve of electric conductivity for undoped TiNTs is very flat while the curve is relatively sharp for the doped ones. After irradiated for $150 \mathrm{~min}$, the electric conductivity for $3 \%$ doped TiNTs is $52 \mu \mathrm{S} / \mathrm{cm}$, which is about 2 -fold higher than that of undoped TiNTs. These results obviously indicate that doping with iron ions is very effective in increasing the photocatalytic activity of TiNTs.

The photocatalytic activity originates from the production of excited electron in the conduction band, along with corresponding positive holes in the valence band through the absorption of suitable illumination [17]. By introducing the $\mathrm{Fe}^{3+}$ in the matrix, $\mathrm{Fe}$ atoms replace the Ti in the crystal lattice, and the oxygen vacancies and defects increase to maintain charge equilibrium. During the process of photocatalytic reaction, oxygen vacancies and defects could become the centers to capture photoinduced electrons so that the recombination of photoinduced electrons and holes was effectively inhibited [26]. Thus, oxygen vacancies and defects were in favor of photocatalytic activity. The oxygen vacancies and defects were increased by the iron doping; consequently, the photocatalytic activity is enhanced by the level of iron doping as shown in Figure 4.

In conclusion, we have firstly reported the synthesis of the iron-doped TiNTs, and this method may be applied to synthesize transition metal ions doped TiNTs and other nanostructures (such as nanobelt). This work provides a facile route to improve the photocatalytic efficiency of materials, and other properties such as magnetic property may also be changed. In addition, existence of Ti-OH on the surface [27] makes the decoration of TiNTs possible, and further assembly may also be achieved. These works are still under research.

\section{Acknowledgment}

Financial support from the National High Technology Research and Development Program of China (863 Program) (Grants 2009AA03Z802 and 2009AA03Z803) is gratefully acknowledged.

\section{References}

[1] P. X. Gao, C. S. Lao, Y. Ding, Z. L. Wang, and Z. L., "Metal/semiconductor core/shell nanodisks and nanotubes," Advanced Functional Materials, vol. 16, pp. 53-62, 2006.

[2] P. Hu, F. Yuan, L. Bai, J. Li, and Y. Chen, "Plasma synthesis of large quantities of zinc oxide nanorods," Journal of Physical Chemistry C, vol. 111, no. 1, pp. 194-200, 2007.

[3] P. Hu, L. Y. Bai, L. J. Yu, J. L. Li, F. L. Yuan, and Y. F. Chen, "Shapecontrolled synthesis of $\mathrm{ZnS}$ nanostructures: a simple and rapid method for one-dimensional materials by plasma," Nanoscale Research Letters, vol. 4, pp. 1047-1053, 2009.

[4] Z. Zhang, C. Shao, P. Zou et al., "In situ assembly of welldispersed gold nanoparticles on electrospun silica nanotubes for catalytic reduction of 4-nitrophenol," Chemical Communications, vol. 47, no. 13, pp. 3906-3908, 2011. 
[5] P. Hu, N. Han, X. Zhang et al., "Fabrication of $\mathrm{ZnO}$ nanorodassembled multishelled hollow spheres and enhanced performance in gas sensor," Journal of Materials Chemistry, vol. 21, no. 37, pp. 14277-14284, 2011.

[6] H. Yu, Z. Zhang, M. Han, X. Hao, and F. Zhu, "A general lowtemperature route for large-scale fabrication of highly oriented $\mathrm{ZnO}$ nanorod/nanotube arrays," Journal of the American Chemical Society, vol. 127, no. 8, pp. 2378-2379, 2005.

[7] P. Hu, X. Zhang, N. Han, W. Xiang, Y. Cao, and F. Yuan, "Solution-controlled self-assembly of $\mathrm{ZnO}$ nanorods into hollow microspheres," Crystal Growth \& Design, vol. 11, no. 5, pp. 1520-1526, 2011.

[8] C. C. Tsai and H. Teng, "Structural features of nanotubes synthesized from naoh treatment on $\mathrm{TiO}_{2}$ with different posttreatments," Chemistry of Materials, vol. 18, pp. 367-373, 2006.

[9] D. Li and Y. Xia, "Fabrication of titania nanofibers by electrospinning," Nano Letters, vol. 3, no. 4, pp. 555-560, 2003.

[10] M. Zhang, Y. Bando, and K. Wada, "Synthesis of coaxial nanotubes: titanium oxide sheathed with silicon oxide," Journal of Materials Research, vol. 16, no. 5, pp. 1408-1412, 2001.

[11] A. Hagfeldt and M. Gratzel, "Light-induced redox reactions in nanocrystalline systems," Chemical Reviews, vol. 95, no. 1, pp. 49-68, 1995.

[12] S. U. M. Khan, M. Al-Shahry, and W. B. Ingler, "Efficient photochemical water splitting by a chemically modified n$\mathrm{TiO}_{2}$," Science, vol. 297, no. 5590, pp. 2243-2245, 2002.

[13] Q. Chen, W. Z. Zhou, G. H. Du, and L. M. Peng, "Trititanate nanotubes made via a single alkali treatment," Advanced Materials, vol. 14, no. 17, pp. 1208-1211, 2002.

[14] A. Thorne, A. Kruth, D. Tunstall, J. T. S. Irvine, and W. Zhou, "Formation, structure, and stability of titanate nanotubes and their proton conductivity," Journal of Physical Chemistry B, vol. 109, no. 12, pp. 5439-5444, 2005.

[15] C. C. Tsai and H. Teng, "Regulation of the physical characteristics of titania nanotube aggregates synthesized from hydrothermal treatment," Chemistry of Materials, vol. 16, no. 22, pp. 4352-4358, 2004.

[16] T. Kasuga, M. Hiramatsu, A. Hoson, T. Sekino, and K. Niihara, "Formation of titanium oxide nanotube," Langmuir, vol. 14, no. 12, pp. 3160-3163, 1998.

[17] X. H. Wang, J. G. Li, H. Kamiyama et al., "Pyrogenic iron(III)doped $\mathrm{TiO}_{2}$ nanopowders synthesized in RF thermal plasma: phase formation, defect structure, band gap, and magnetic properties," Journal of the American Chemical Society, vol. 127, pp. 10982-10990, 2005.

[18] R. Asahi, T. Morikawa, T. Ohwaki, K. Aoki, and Y. Taga, "Visible-light photocatalysis in nitrogen-doped titanium oxides," Science, vol. 293, no. 5528, pp. 269-271, 2001.

[19] H. Irie, Y. Watanabe, and K. Hashimoto, "Nitrogenconcentration dependence on photocatalytic activity of $\mathrm{TiO}_{2-x} \mathrm{~N}_{x}$ powders," Journal of Physical Chemistry B, vol. 107, no. 23, pp. 5483-5486, 2003.

[20] B. C. Cheng, Y. H. Xiao, G. S. Wu, and L. D. Zhang, "Controlled growth and properties of one-dimensional $\mathrm{ZnO}$ nanostructures with Ce as activator/dopant," Advanced Functional Materials, vol. 14, no. 9, pp. 913-919, 2004.

[21] B. D. Yuhas, D. O. Zitoun, P. J. Pauzauskie, R. R. He, and P. D. Yang, "Transition-metal doped zinc oxide nanowires," Angewandte Chemie International Edition, vol. 45, no. 3, pp. 420-423, 2006.
[22] J. H. He, C. S. Lao, L. J. Chen, D. Davidovic, and Z. L. Wang, "Large-scale Ni-doped $\mathrm{ZnO}$ nanowire arrays and electrical and optical properties," Journal of the American Chemical Society, vol. 127, no. 47, pp. 16376-16377, 2005.

[23] M. A. Khan, H. T. Jung, and O. B. Yang, "Synthesis and characterization of ultrahigh crystalline $\mathrm{TiO}_{2}$ nanotubes," Journal of Physical Chemistry B, vol. 110, no. 13, pp. 6626-6630, 2006.

[24] J. Frank, J. V. Geil, and R. Freaso, "Automatic determination of oxidation stability of oil and fatty products [Food quality control, vegetable and animal fats]," Food Technology, vol. 36, no. 6, pp. 71-76, 1982.

[25] M. K. Läubli and P. A. Bruttel, "Determination of the oxidative stability of fats and oils: comparison between the active oxygen method (AOCS Cd 12-57) and the rancimat method," Journal of the American Oil Chemists' Society, vol. 63, no. 6, pp. 792-795, 1986.

[26] L. Q. Jing, X. J. Sun, B. F. Xin, B. Q. Wang, W. M. Cai, and H. $\mathrm{G}$. Fu, "The preparation and characterization of la doped $\mathrm{TiO}_{2}$ nanoparticles and their photocatalytic activity," Journal of Solid State Chemistry, vol. 177, no. 10, pp. 3375-3382, 2004.

[27] X. T. Zhang, Y. M. Wang, C. M. Zhang et al., "Chemical modified titanate nanotubes and their stable luminescent properties," Science in China Series B, vol. 35, pp. 1-6, 2005. 

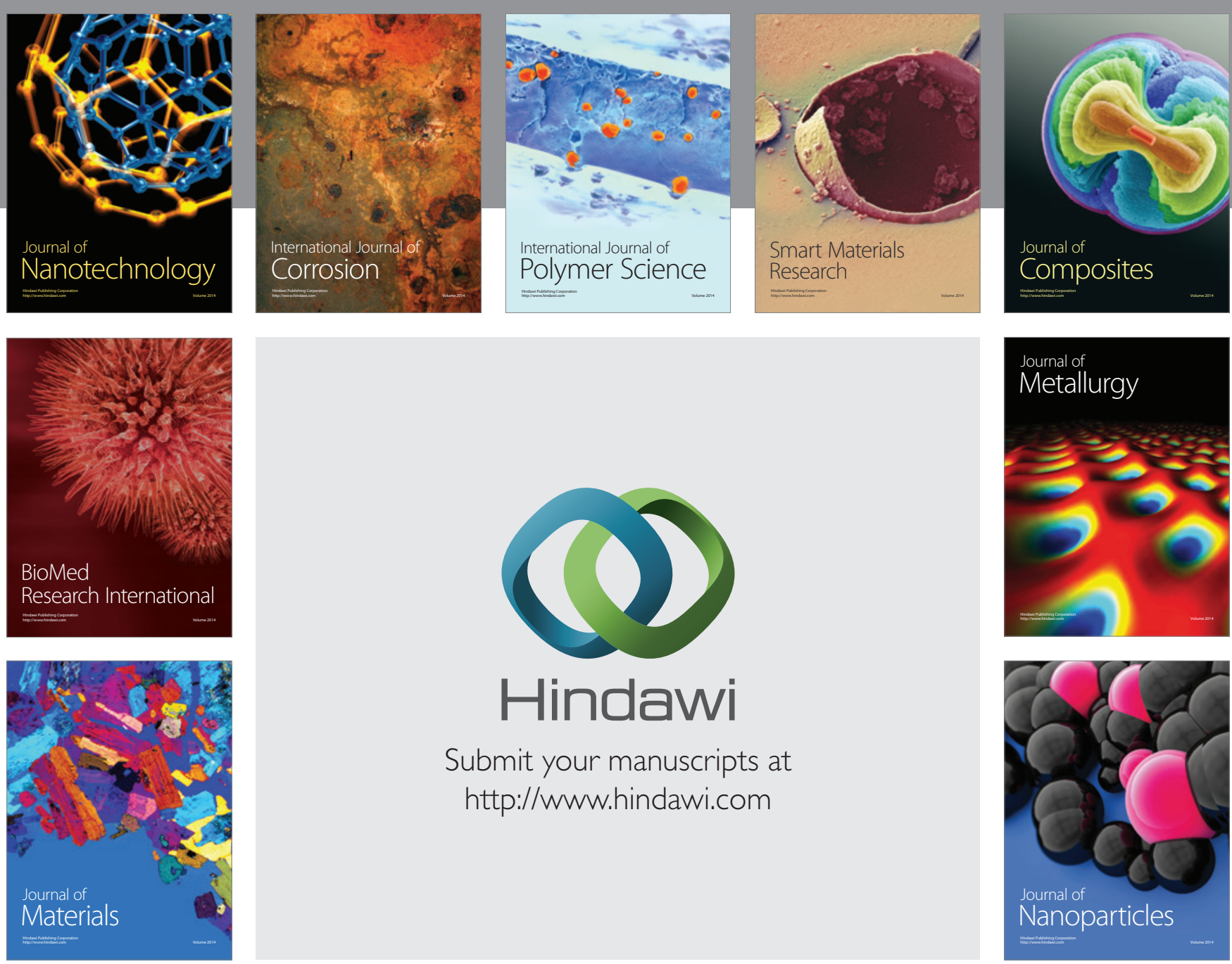

Submit your manuscripts at http://www.hindawi.com
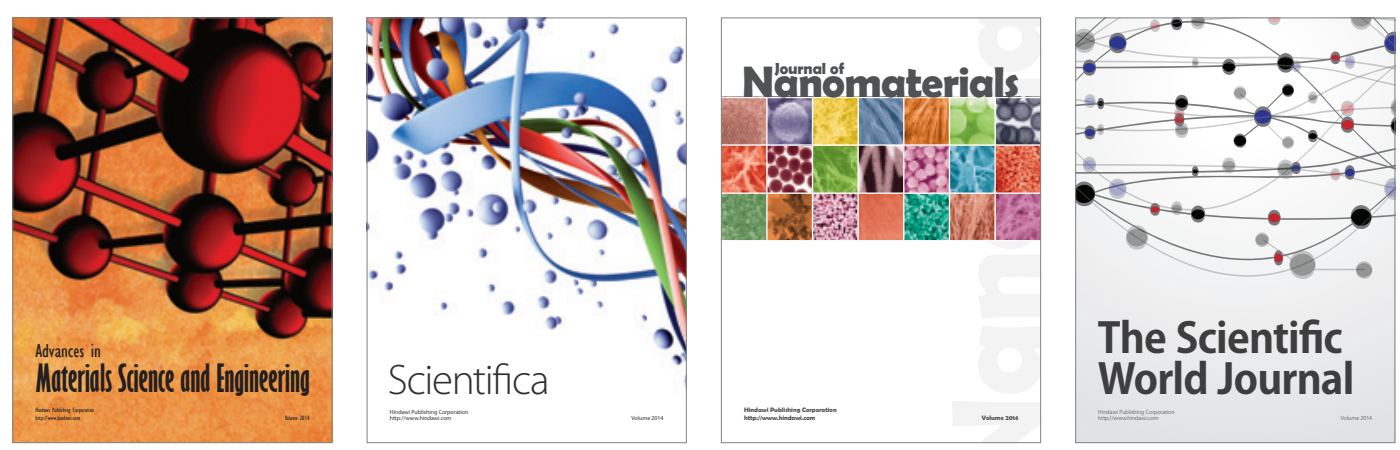

\section{The Scientific World Journal}
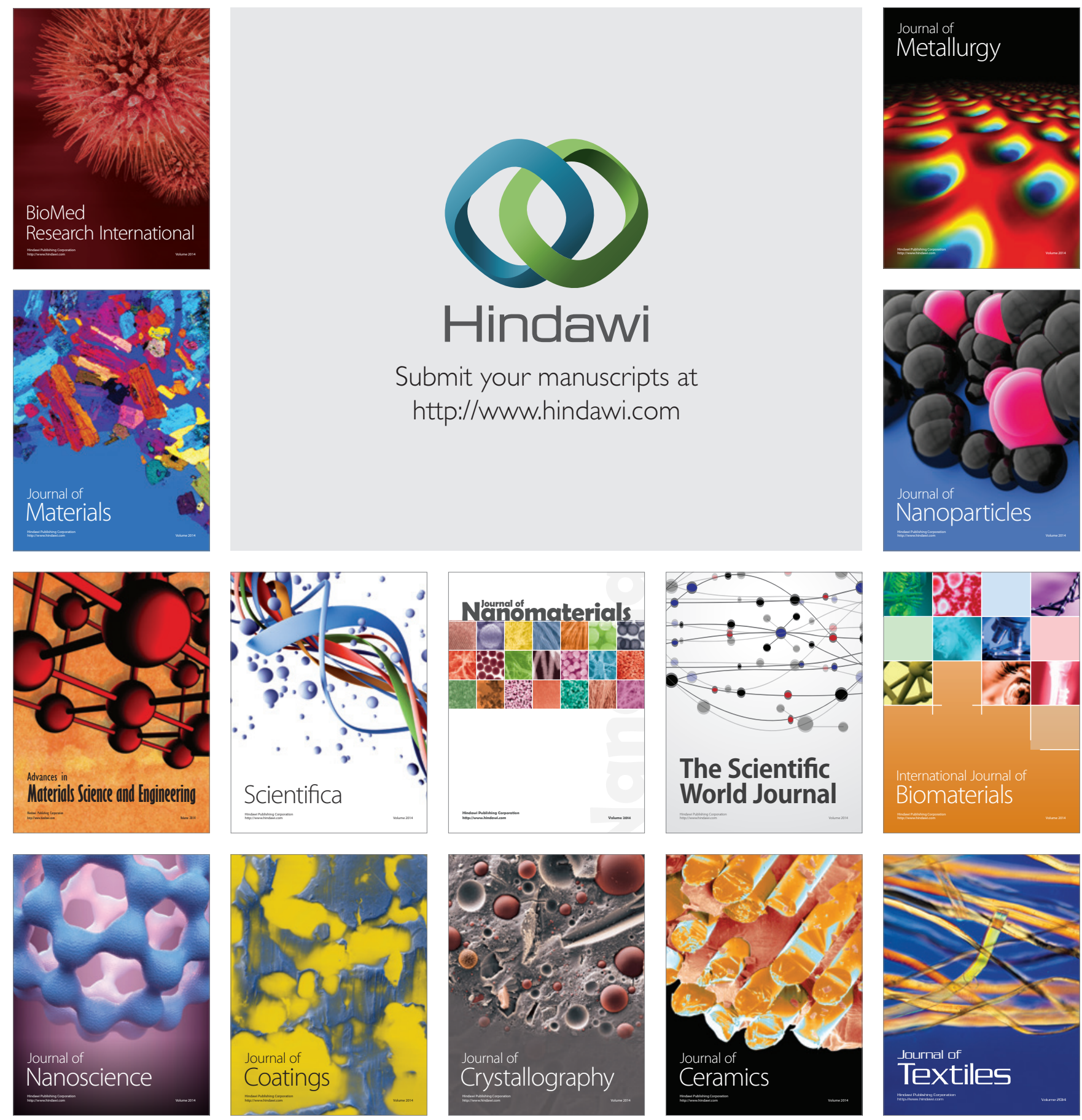